\title{
Reliability Assessment for the Braking System of EMUs Based on Small Sample Size and GO Method
}

\author{
Zhao Chunqing ${ }^{1,2}$, Yang Jianwei ${ }^{1,2,}$, Liu Haibo ${ }^{3}$, Wang Wenwen ${ }^{1,2}$ and Qian Ying ${ }^{1,2}$ \\ ${ }^{1}$ School of Machine-Electricity and Automobile Engineering, Beijing University of Civil Engineering and Architecture, \\ Beijing, 100044, China \\ ${ }^{2}$ Beijing Engineering Research Center of Monitoring for Construction Safety, Beijing University of Civil Engineering \\ and Architecture, Beijing, 100044, China; \\ ${ }^{3}$ CSR Qingdao Sifang CO., LTD. Qingdao, 266111, China
}

\begin{abstract}
In order to solve the reliability evaluation problem of the braking system of EMUs, the GO-ss method has been proposed in this paper. In the proposed method, multi-Bayes theory is applied to analyze the failure rate of key component brake controller under small sample. And then, the braking system is assessed based on GO method to achieve the reliability assessment of EMUs brake control system. Finally, the results of experiment and test data validate that this method can solve the reliability evaluation problem of the braking system, which provides a theoretical basis for the safe operation of the braking system of EMUs.
\end{abstract}

Keywords: Braking system, EMUs, GO method, multi-Bayes theory, small sample.

\section{INTRODUCTION}

The reliability evaluation of the high-speed train, which is one important transport means, has attracted more and more attention. Based on the Bayes theory, reference [1] makes the reliability assessment for high-speed train bearing under extreme sample size which solves the reliability life problem of the bearing. According to the Bayes theory, reference [2] assesses the reliability of key structure of C70 gondola car which follows Gaussian distribution under extreme sample condition. However, there is seldom assessment of reliability of electronic components, and the distributions and methods have certain limitations. In addition, for metro traction power system, the reference [3] does reliability research based on the GO method, and the results prove that the GO method can accurately reflect reliability of the system. On the other hand, GO method is applied to the reliability analysis of EMUs pouring system in reference [4], which shows that the GO method is convenient and concise with high precision. But numerous studies only evaluate the reliability of the components or system.

For high-speed EMUs, the braking system is particularly important. Although the EMUs braking system components are products with high reliability, they will lead to serious consequences once the failure occurs. The common faults of EMUs braking system mainly include: brake control device failure, lack of braking force, monitor display locking, low total air pressure, etc. Therefore, it is necessary to assess reliability of the braking system. To solve the reliability problem of the braking system, this paper puts

*Address correspondence to this author at the No. 1 Zhanlanguan Road., Xicheng District, Beijing, Postcard: 100044, China; Tel: 01068322515 ;

E-mail: yangjianwei@bucea.edu.cn forward the GO-ss method combining small sample with GO method on the basis of others' research.

\section{MATERIALS AND METHODOLOGY}

\subsection{The Reliability Model of EMUs Brake Control Sys- tem}

Firstly, under small sample condition, the brake controller, which is the key component of the braking system with high reliability, is assessed based on the Bayes theory. And then the reliability of braking system is assessed based on the GO method, which provides a basis for the safe operation of the system.

\section{(1) The EMUs Brake Control System}

EMUs brake device includes the regenerative brake device and electric commanding air brake device. Trailer cars adopt full air brake mode. Under the control of the drivers and other control devices, the brake control system produces and transmits brake signals and assigns braking ratio to different brake modes. Brake control device is composed of brake controller, all kinds of air brake valves and the air cylinder, which are hanging below the floor of the vehicle as a whole component. Brake control device controls the braking actions including the service braking, quick-acting braking, emergency braking and snow resistance braking [5].

The control principle of the braking system is as follows: the driver brake controller sends out the braking instruction which is latter transmitted to the brake control device of every car through the information monitoring system of the train, and then the BCU calculates necessary air braking force. According to the braking control rule, the brake controller outputs the control current which is converted to air pressure by EP value. After the pressure is 


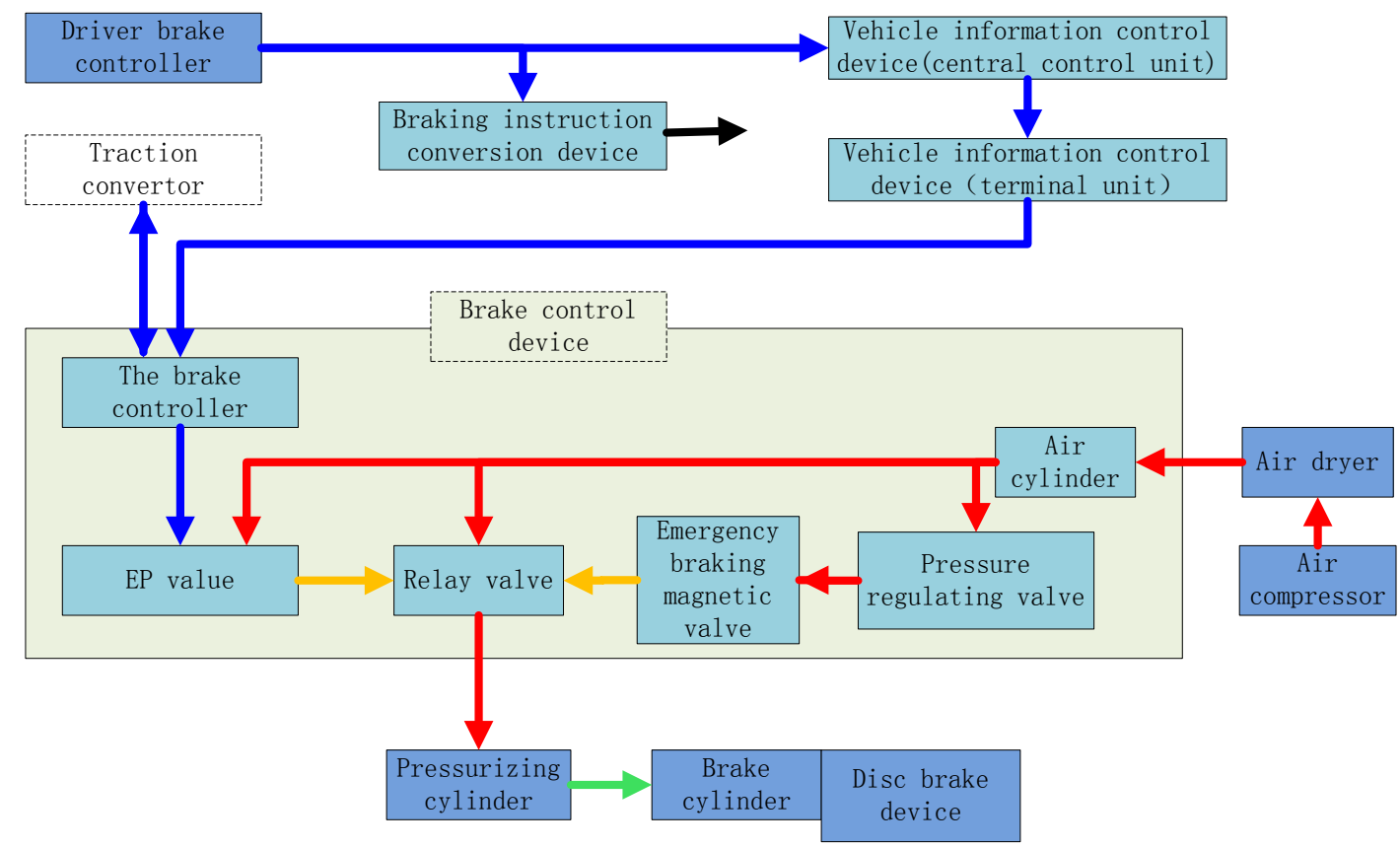

Fig. (1). The brake control principle of EMUs.

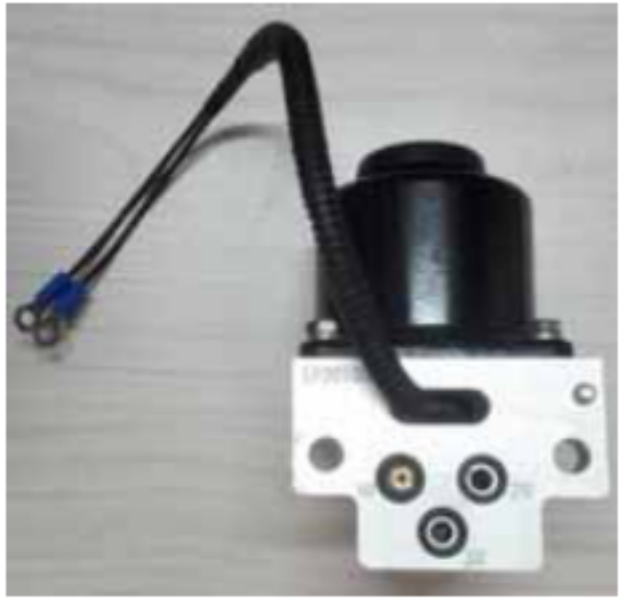

Fig. (2). The physical diagram of EP valve.

magnified by the relay valve, the compressed air is sent to pressurizing cylinder to make the basic brake equipment complete the braking action. The brake control principle of EMUs is shown in Fig. (1). where arrows represent different meanings and colors: blue, red, green and yellow respectively mean electric instructions, compressed air, air instructions and oil.

The brake controller, which is brake control unit (BCU) setting in the brake control device, adapts microprocessor digital processing method. Braking instruction from braking test-bed gets through the center device and transmission terminal using optical cable, and then BCU calculates the braking force according to the load signal and speed signal of each compartment to control electric braking force and air braking force.

The electro-pneumatic changeover valve (EP value) belongs to a kind of the control valves. Its role is to convert current instructions corresponding to braking force from BCU to air pressure. At the same time, the air pressure can continuously vary due to the control of electromagnetic department. As a control signal, the pressure controls air supply and exhaust of the relay valve. The physical diagram of EP valve is shown in Fig. (2), which is made of electromagnetic department, gas supply department and exhaust department as shown in Fig. (3).

(2) Reliability model of the braking system

i) Reliability model under small sample condition

Under small sample condition, the classical statistical methods cannot meet the requirements in the aspect of the use of a priori information and the comprehensive application of experiment multi-data due to low amount of information. So, the Bayes method is often used to solve reliability problem of complex systems under small sample in the actual reliability engineering, which can get realistic 


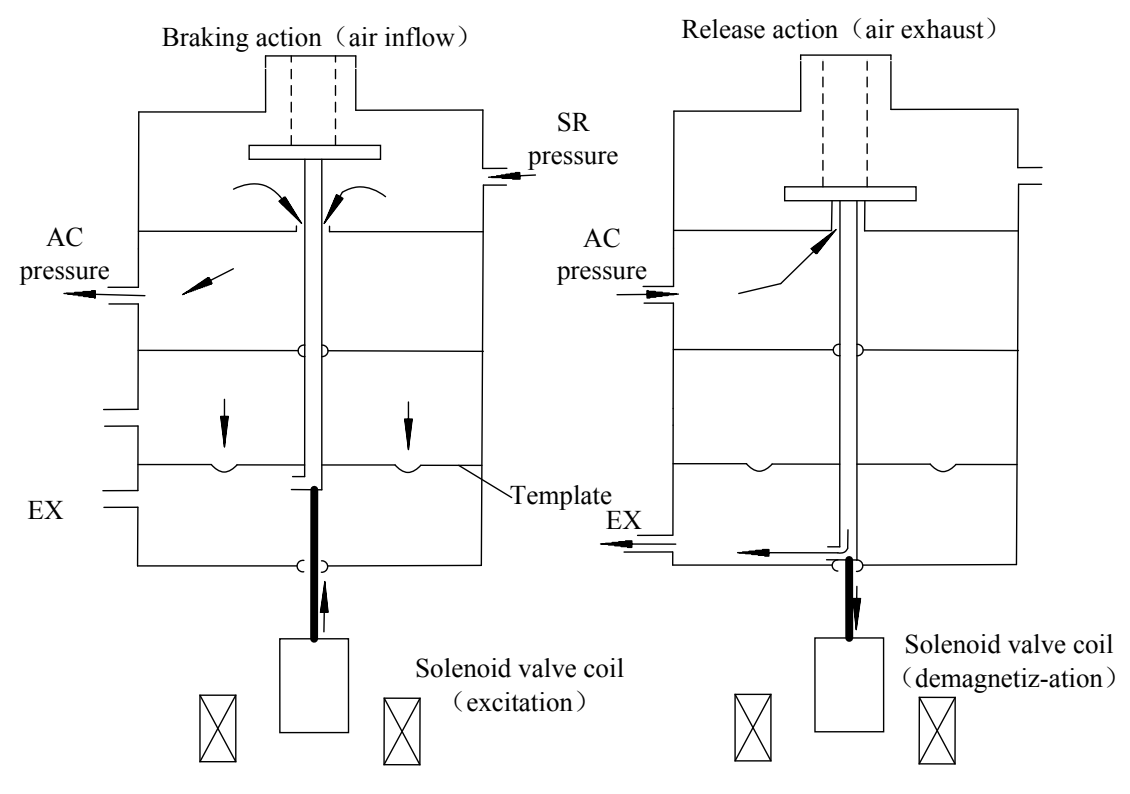

(a)

(b)

(a) Braking action (air inflow)

(b) Release action(air exhaust)

Fig. (3). The schematic of EP value.

subjective probability by making full use of the historical information and the existing information.

In addition, in the reliability theory, the exponential distribution is life distribution of products at random failure stage, especially for electronic products, which is one of the most basic and common distributions. For products with high reliability and long life, the structure is complicated and price can be costly which means that it is impossible to process the samples for field test for a long time, so the research on reliability test and evaluation under small sample size is particularly important. Sometimes due to the limited time and actual experimental condition, we often assess reliability using time truncations censoring test during the reliability testing. In time truncations censoring test, the test data received by products with high reliability and long life is called zero-failure data when failures do not occur.

Starting from the unit-level components, we assume the model of the component as follows: in time truncations censoring test including $\mathrm{m}$ times, censored time is $t_{i}$ $(i=1,2, \cdots, m)$, where, $t_{1}<t_{2}<, \cdots,<t_{m}$; the corresponding number of test samples is $n_{i}$, and the experimental results $\left(t_{i}, n_{i}\right)$ are zero-failure data. We suppose that the life of product $\mathrm{T}$ obeys exponential distribution of parameter $\lambda$, and the failure density of product is given as follows:

$$
f(t)=\lambda e^{-\lambda t} \mathrm{t} \geq 0, \lambda>0
$$

On the basis of zero-failure data, we introduced failure information and realized multi-Bayes estimation to make comprehensive research on product [6]. Thus, the evaluation of the failure rate of the brake controller is achieved under small sample condition.

ii) GO method model
The GO method is a kind effective method of system reliability analysis. The GO method uses GO diagram to simulate system, and GO diagram is similar to the system diagram in which signal flow corresponds to the logistics of system. The basic idea of the GO method is that system principle diagrams, flow charts or engineering drawings are translated into GO diagrams. The main steps of the GO method are to establish GO diagram and GO operation, and two elements of GO diagram and GO operation are operator and signal flow. The component or subsystem of system are referred to as units, and the operator represents the logical relationship between elementary function and input and output signal. GO method has defined 17 kinds of standard operators. The properties of operators include types, data and operation rules, and the type contains the unit function and feature, which is the main property of operator [7].

As shown in Fig. (4), it's the logical schematic of highspeed EMUs brake control device, in which the meaning of arrows in different colors is the same as Fig. (1). In the system, type 1 operator simulates air dryer, air cylinder, regulating valve, emergency braking magnetic valve and brake cylinder and other mechanical transmission components; type 5 operator simulates the brake controller, air compressor and other input units; type 6 operator simulates EP valve; and type 10 operator simulates relay valve. Thus, the logical schematic is translated into GO graph model to study the brake control process of EMUs, as shown in Fig. (5). In this figure, the left side of the hyphen respectively represents the type of operator, and the right side respectively, the corresponding number of the system unit [7]. The contents of unit number are shown in Table 3 . 


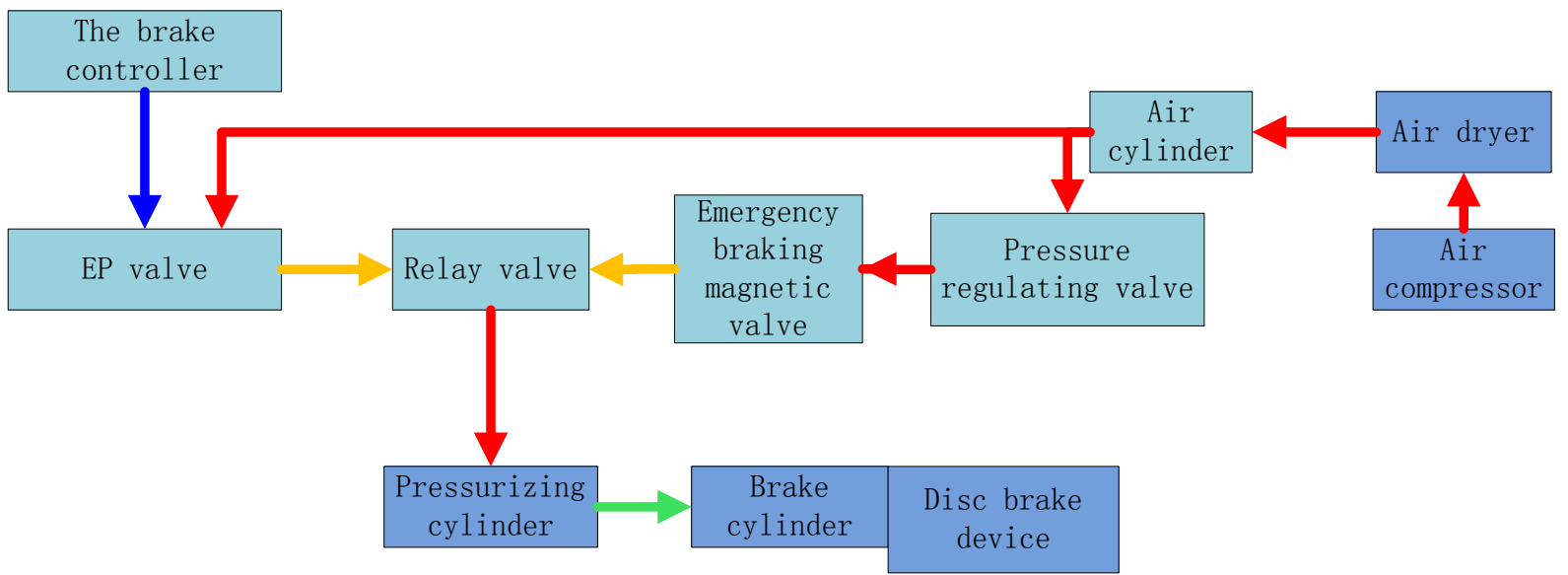

Fig. (4). Logic schematic of brake control device.

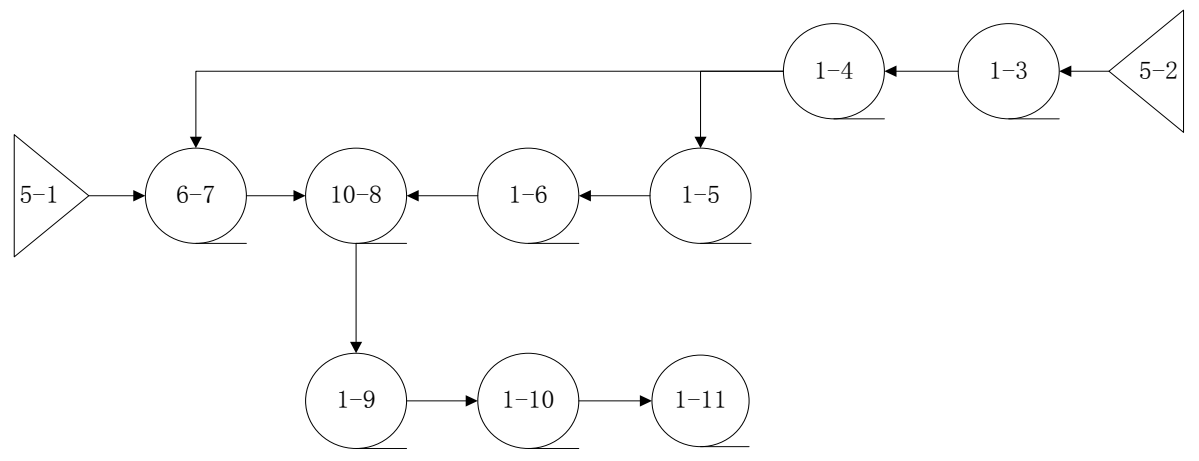

Fig. (5). GO method model of brake control device.

\subsection{Reliability Assessment Based on Small Sample Size and GO Method}

(1) Reliability assessment based on the Bayes theory under small sample size

i) The selection of the prior distribution

The key to the Bayes method is how to choose the prior distribution. There are many ways to structure a priori distribution, such as: Maximum Entropy Method (MEM), the conjugate distribution method, the Bootstrap method, using the previous test data and empirical Bayes method, etc. The conjugate prior distribution, which is put forward in the $1960 \mathrm{~s}$, is often selected as the prior distribution of Bayes method, through which posterior distribution can be easily gotten. Based on the Bayes method, this paper selects the conjugate priori Gamma distribution as prior distribution of failure rate for reliability evaluation of product whose life obeys exponential distribution. The failure density function is given as follows:

$\pi(\lambda \mid a, b)=\frac{b^{a}}{\Gamma(a)} \lambda^{a-1} e^{-b \lambda}$

where, the shape parameter $\mathrm{a}>0$, the scale parameter $\mathrm{b}>$ $0, \Gamma(a)=\int_{0}^{\infty} x^{\alpha-1} e^{-x} d x$.

The first derivative about $\lambda$ of $\pi(\lambda \mid a, b)$ : $\dot{\pi}(\lambda \mid a, b)=\frac{b^{a}}{\Gamma(a)} \lambda^{a-2} e^{-b \lambda}(a-1)(-b)$

where, $a>0, b>0$.

In the case of zero-failure data [8], we select $0<\mathrm{a} \leq 1$, $\mathrm{b}>0$, which can make the density function $\pi(\lambda \mid a, b)$ decrease with increased $\lambda$. The selection of $b$ cannot be too big according to the robustness of Bayes estimation. Based on experts' experience, we choose $\mathrm{b}$ up to $5 \sim 9$, which is set to $\mathrm{c}$, and we choose $0<\mathrm{a} \leq 1, \quad 0<\mathrm{b}<\mathrm{c}$.

When priori information is not enough, hyperparameter is difficult to determine, so we use multi-Bayes method to determine the prior distribution of parameter. Based on the idea of multi-Bayes method, we take the hyper-parameter $\mathrm{b}$ as random variable, and its distribution $\pi(b)$ is a super-prior distribution. And then, we can choose a suitable non-informative prior distribution.

When $\mathrm{a}=1, \pi(\lambda \mid a, b)$ is exponential distribution function, and the multi-prior distribution function is obtained as follows:

$\pi(\lambda \mid 1, b)=\frac{b}{\Gamma(1)} e^{-b \lambda}, b>0$

In addition, we select subjectively uniform distribution on $(0, c)$ as prior distribution of $b$. So, the density function of parameter $b$ is as follows: 
$\pi(b)=\frac{1}{c}, 0<b<c$

Based on the multi-prior distribution[6], the failure density is obtained as follows:

$\pi(\lambda)=\int_{0}^{c} \pi(\lambda \mid 1, b) \pi(b) d b=\frac{1}{c} \int_{0}^{c} b \exp (-b \lambda) d b$

ii) The Bayes estimation of failure rate

Ming Han (reference [9]) studied about products whose life follows exponential distribution, and conducted time truncations censoring test. Among $m$ times of time truncations censoring test, let us suppose that the number of failure sample in the $\mathrm{i}$-th truncation test is $\mathrm{x}_{i}$ which follows Poison distribution of parameter $\lambda_{i} t_{i} n_{t}$ as follows:

$P\left(x_{i}=r_{i}\right)=\frac{\left(\lambda t_{i} n_{i}\right)^{r_{i}}}{\left(r_{i}\right) !} \exp \left(-\lambda t_{i} n_{i}\right), r=1,2, \cdots, n_{m+1}, i=1,2, \cdots, m$

And the likelihood function of the failure rate is given as follows:

$L(\lambda \mid \mathrm{x})=\prod_{i=1}^{m} p_{i}\left(x_{i}=r_{i}\right)=\left[\prod_{i=1}^{m} \frac{\left(\lambda t_{i} n_{i}\right)^{r_{i}}}{\left(r_{i}\right) !}\right] \exp (-M \lambda)$

When the result of $\mathrm{m}$ times test is zero-failure data $\left(\mathrm{t}_{\mathrm{i}} n_{i}\right)$, the likelihood function of the failure rate is obtained as follows:

$$
\begin{aligned}
& L(\lambda)=\exp (-M \lambda) \\
& \text { where, } M=\sum_{\mathrm{i}=1}^{\mathrm{m}} \mathrm{t}_{\mathrm{i}} n_{i} .
\end{aligned}
$$

According to the Bayes formula, the posterior density function of $\lambda$ is obtained as follows:

$$
h(\lambda \mid M)=\frac{\pi(\lambda) L(\lambda \mid 0)}{\int_{0}^{\infty} \pi(\lambda) L(\lambda \mid 0) d \lambda}=\frac{\exp (-M \lambda) \int_{0}^{c} \exp (-b \lambda) d b}{c-M \ln \left(\frac{c+M}{M}\right)}
$$

Under the condition of the given posterior distribution and loss function, we can get the Bayes estimation of parameter. And based on quadratic loss function, multi-Bayes estimation of parameter $\lambda$ can be obtained as follows:

$\hat{\lambda}_{1}=\int_{0}^{\infty} \lambda h(\lambda \mid M) d \lambda=\left[\ln \left(\frac{c+M}{M}\right)-\frac{c}{M+c}\right] /\left[c-M \ln \left(\frac{c+M}{M}\right)\right]$

3) The estimation of failure rate by introducing the failure information

Ming Han conducts time truncations censoring test for $\mathrm{m}+1$ times for product obeying exponential distribution $[10,11]$, among which the data of the first $m$ times is zerofailure data, and the $(m+1)$ th time contains failure data. We set that the test sample number of the $(m+1)$ th test equals to $\mathrm{n}_{m+1}$, the truncation time equals to $t_{m+1}+1$, and the failure sample number equals to $r$, where, $r=0,1,2, \cdots, n_{m+1}$. At the same time, we suppose that there is one failure sample, and according to reference $[12,13], t_{m+1}$ is obtained as follows:

$n_{m+1}=\left[\frac{1}{m} \sum_{i=1}^{m} n_{i}\right], t_{m+1}=t_{m}+\frac{1}{\lambda_{1}}, r=1$
Where, $[\mathrm{x}]$ is an integer and less than or equal to $\mathrm{x}$.

At the same time, based on the quadratic loss function, the multi-Bayes estimation of parameter $\lambda$ by introducing the failure information can be obtained as follows:

$$
\hat{\lambda}_{2}=(r+1) \frac{\int_{0}^{c} \frac{b}{(N+b)^{r+2}} d b}{\int_{0}^{c} \frac{b}{(N+b)^{r+1}} d b}
$$

where, $N=\sum_{i=1}^{m+1} t_{i} n_{i}$.

Analyze the reliability of product by synthesizing zerofailure data of the first $m$ times and failure information of the $(m+1)$ th [8]. And according to the weighted average of $\lambda_{1}$ and $\lambda_{2}$, the synthetic estimation is obtained as follows:

$\hat{\lambda}=\frac{1}{N}\left\{M * \hat{\lambda}_{1}+\left(\mathrm{t}_{m+1} n_{m+1}\right) * \hat{\lambda}_{2}\right\}$

Therefore, MTBF can be obtained as follows:

$$
\hat{\operatorname{MTB} F}=\frac{1}{\hat{\lambda}}
$$

(2) The reliability assessment based on the GO method

The repairable system has two kinds of state: the good working state and breakdown maintenance state, which randomly appear in the whole life of product. The GO method can not only analyze the probability when the system works, but also the failure rate. Depending on reference [7], the reliability characteristics of the system are as follows:

i) Failure Rate $\lambda$;

ii) Maintenance rate $\mu$;

ii) Mean time between failure and maintenance:

$\mathrm{MTBF}=\frac{1}{\lambda}$

iv) Mean time to repair:

$\operatorname{MTTR}=\frac{1}{\mu}$

v) The mean life cycle:

$M C T=M T B F+M T T R$

vi) Mean availability:

$A=\frac{M T B F}{M C T}=\frac{\mu}{\lambda+\mu}$

vii) Mean unavailability: 
Table 1. Zero-failure data of brake controller.

\begin{tabular}{|c|c|c|c|c|c|c|c|c|}
\hline $\mathbf{I}$ & $\mathbf{1}$ & $\mathbf{2}$ & $\mathbf{3}$ & $\mathbf{4}$ & $\mathbf{5}$ & $\mathbf{6}$ & $\mathbf{7}$ & $\mathbf{8}$ \\
\hline \hline $\mathrm{t}_{i}$ & 100 & 200 & 500 & 700 & 800 & 900 & 1000 & 1100 \\
\hline $\mathrm{n}_{i}$ & 4 & 4 & 3 & 3 & 2 & 2 & 1 \\
\hline
\end{tabular}

Table 2. The result data of $\lambda_{1}$.

\begin{tabular}{|c|c|c|c|c|c|c|}
\hline $\mathbf{c}$ & $\mathbf{5}$ & $\mathbf{6}$ & $\mathbf{7}$ & $\mathbf{8}$ & $\mathbf{9}$ & Range \\
\hline \hline$\hat{\lambda}_{1} \times 10^{-5}$ & 9.7056 & 9.7050 & 9.7043 & 9.7037 & 9.7031 & 0.0025 \\
\hline
\end{tabular}

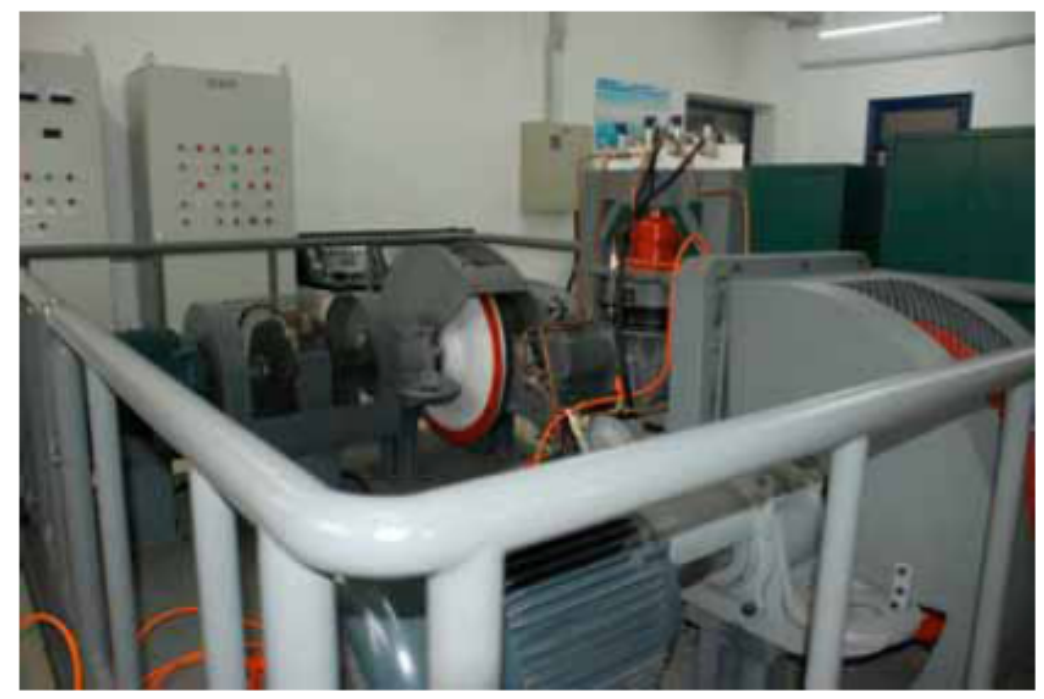

Fig. (6). Data acquisition and processing test bed of the brake controller.

$$
\bar{A}=1-A=\frac{M T T R}{M C T}=\frac{\lambda}{\lambda+\mu}
$$

8) Mean failure times in unit time:

$$
f=A \lambda=\bar{A} \mu=\frac{1}{M C T}
$$

\subsection{The Reliability Analysis of System Based on Experi- mental Data}

(1) The failure rate based on Bayes theory under small sample

The brake control device on each EMU vehicle is composed of brake control unit (referred to as BCU, or called the brake controller), EP valve, relay valve, emergency braking magnetic valve, etc. The brake controller is one of the important parts of brake control device. It receives common braking command or rapid braking command from fiber-optical and wires, and combines speed, air spring pressure, regenerative braking force so that it can calculate the air braking force required for controlling the output current [5]. Its performance will directly affect the operation reliability and safety of the train. Data acquisi- tion and processing test bed of the brake controller is shown in Fig. (6).

We assume that the life of brake controller follows exponential distribution. Now, we have a group of zerofailure data of brake controller, which contains 20 samples, and conducts 8 times truncation censoring test. Zero-failure data is shown in Table $\mathbf{1}$, where, the unit of time is second and $5 \leq \mathrm{c} \leq 9$.

From Table 1, M is obtained as follows:

$M=\sum_{\mathrm{i}=1}^{8} \mathrm{t}_{\mathrm{i}} n_{i}=10300$

And then the failure rate $\lambda_{1}$ can be obtained in Table 2 .

From the result data of Table 2 , the range of $\lambda_{1}$ can be obtained, which shows that compared to $\lambda_{1}, \mathrm{c}$ is steady. We take $\mathrm{c}=5$. According to the above, the failure data is introduced as follows:

$$
t_{m+1}=t_{m}+\frac{1}{\hat{\lambda}}=1.1403
$$


Table 3. Each unit and characteristic data of GO method model of brake control system.

\begin{tabular}{|c|c|c|c|}
\hline Operator number & Unit or logic gates & Failure rate $/ \mathrm{h}^{-1}$ & MTTR/h \\
\hline 1 & Brake controller & $5.1002 \mathrm{E}-5$ & 0.5000 \\
\hline 2 & Air compressor & $4.9736 \mathrm{E}-5$ & 2.6017 \\
\hline 3 & Air dryer & $2.7593 \mathrm{E}-5$ & 5.5784 \\
\hline 4 & Air cylinder & $6.3754 \mathrm{E}-6$ & 5.0186 \\
\hline 5 & Pressure regulating valve & $1.0854 \mathrm{E}-5$ & 4.9054 \\
\hline 6 & Emergency braking magnetic valve & $1.8060 \mathrm{E}-5$ & 0.1202 \\
\hline 7 & EP value & $1.8090 \mathrm{E}-5$ & 0.5131 \\
\hline 8 & Relay valve & $1.8090 \mathrm{E}-5$ & 0.5141 \\
\hline 9 & Pressurizing cylinder & $6.3864 \mathrm{E}-6$ & 5.0214 \\
\hline 10 & Brake cylinder & $6.3867 \mathrm{E}-6$ & 1.1900 \\
\hline 11 & Disc brake device & $1.0412 \mathrm{E}-4$ & 1.7354 \\
\hline
\end{tabular}

$$
n_{m+1}=\left[\frac{1}{m} \sum_{i=1}^{m} n_{i}\right]=[1 / 8 \times 20]=2
$$$$
\mathrm{r}=1
$$

On the basis of Eq. (13), the estimation of $\lambda$ introducing the failure information is obtained as follows:

$$
\hat{\lambda}_{2}=(r+1) \frac{\int_{0}^{c} \frac{b}{(N+b)^{r+2}} d b}{\int_{0}^{c} \frac{b}{(N+b)^{r+1}} d b}=3.0203 \times 10^{-5}
$$

Then, we can obtain the result as follows:

$$
\begin{aligned}
& \hat{\lambda}=\frac{1}{N}\left\{M * \hat{\lambda}_{1}+\left(t_{m+1} n_{m+1}\right) * \hat{\lambda}_{2}\right\}=5.1002 \times 10^{-5} \\
& \hat{M T B F}=\frac{1}{\hat{\lambda}}=1.9607 \times 10^{4}
\end{aligned}
$$

2) The logic simplifying operation of system GO model

According to the estimator of the failure rate based on the Bayes theory under the small sample above, we have obtained the failure rate of brake controller, and the reliability of the rest of unit can be received from historical data and experience data. The data is shown in Table $\mathbf{3}$, in which the content of the unit number corresponds to the content in Fig. (3).

In this paper, we assumed that each unit of braking system is a repairable system of steady-state analysis with two sates: the normal state and the fault state, having the features of normal state probability, fault state probability, failure rate, repair rate of input signal of operator, operator and output signal of operator are expressed respectively as follows: $\left[\mathrm{P}_{\mathrm{S}(1)}, \mathrm{P}_{\mathrm{S}(2)}, \lambda_{\mathrm{S}}, \mu_{\mathrm{S}}\right],\left[\mathrm{P}_{\mathrm{C}(1)}, \mathrm{P}_{\mathrm{C}(2)}, \lambda_{\mathrm{C}}, \mu_{\mathrm{C}}\right]$ and $\left[\mathrm{P}_{\mathrm{R}(1)}, \mathrm{P}_{\mathrm{R}(2)}, \lambda_{\mathrm{R}}, \mu_{\mathrm{R}}\right]$ [9]. In the $\mathrm{GO}$ figure, we processed operators which are completely in series with the logic as a whole module, and the related failure rate is obtained through simple addition operation, meanwhile maintenance rate can be also calculated through partial sum. For shared signals, the result will be wrong if output eigenvalue is repeated in calculation, so the deviation brought by shared signals needs to be modified.

In the GO model of Fig. (5), the units including operators $2 \sim 4,4 \sim 6,1$ and 7 are treated as a complete series module, and the calculation methods for the characteristics are given as follows:

$\lambda_{R 4}=\sum_{i=2}^{4} \lambda_{C i}$

$\mu_{R 4}=\frac{\lambda_{R 4}}{\sum_{i=2}^{4} \frac{\lambda_{C i}}{\mu_{C i}}}$

$\lambda_{R 6}=\sum_{i=5}^{6} \lambda_{C i}+\lambda_{R 4}$ 
Table 4. System reliability analysis results.

\begin{tabular}{|c|c|}
\hline System reliability analysis eigenvalue & Value \\
\hline \hline$\lambda /\left(10^{4} h\right)^{-1}$ & 2.08501 \\
\hline$\mu /\left(10^{4} h\right)^{-1}$ & 6883.8615 \\
\hline$M T B F / h$ & 4796.1309 \\
\hline$M T T R / h$ & 1.4527 \\
\hline$M C T / h$ & 4797.5835 \\
\hline$A$ & 0.9997 \\
\hline $\bar{A}$ & 0.0003028 \\
\hline$f /\left(\right.$ frequence $\left.\bullet\left(10^{4} \mathrm{~h}\right)^{-1}\right)$ & 2.0844 \\
\hline
\end{tabular}

$$
\mu_{R 6}=\frac{\lambda_{R 6}}{\sum_{i=5}^{6} \frac{\lambda_{C i}}{\mu_{C i}}+\frac{\lambda_{R 4}}{\mu_{R 4}}}
$$

$\lambda_{R 7}=\lambda_{C 1}+\lambda_{R 4}+\lambda_{C 7}$

$$
\mu_{R 7}=\frac{\lambda_{R 7}}{\frac{\lambda_{C 1}}{\mu_{C 1}}+\frac{\lambda_{R 4}}{\mu_{R 4}}+\frac{\lambda_{C 7}}{\mu_{C 7}}}
$$

The input of operator 8 has used the output of operators 6 and 7, but the input of operators 6 and 7 all includes the output of operator 4 which leads to repeated calculation of the output of operator 4 . So, the deviation needs to be modified as follows:

$$
\lambda_{R 8}=\lambda_{R 6}+\lambda_{R 7}-\lambda_{R 4}
$$

$$
\mu_{R 8}=\frac{\lambda_{R 8} P_{R 8}(1)}{1-P_{R 8}(1)}=\lambda_{R 8} \frac{\frac{P_{R 6}(1) P_{R 7}(1)}{P_{R 4}(1)}}{1-\frac{P_{R 6}(1) P_{R 7}(1)}{P_{R 4}(1)}}
$$

where, $P_{R \mathrm{i}}(1)=\mu_{R i} /\left(\lambda_{R \mathrm{i}}+\mu_{R i}\right), P_{R \mathrm{i}}(2)=1-P_{R \mathrm{i}}(1)$.

Similarly, the units including operators $8 \sim 11$ are a complete series module, and the characteristic calculation methods are obtained as follows:

$$
\begin{aligned}
& \lambda_{R 11}=\sum_{i=9}^{11} \lambda_{C i}+\lambda_{R 8} \\
& \mu_{R 11}=\frac{\lambda_{R 11}}{\sum_{i=9}^{11} \frac{\lambda_{C i}}{\mu_{C i}}+\frac{\lambda_{R 8}}{\mu_{R 8}}}
\end{aligned}
$$

\section{RESULTS}

From Eqs. (16)-(21) and Eqs. (22)-(31), the reliability analysis data of brake control system is shown in Table 4.

\section{CONCLUSION}

The reliability analysis data of brake control system is shown in Table 4. The MTBF for a EMU of brake control device is $4900 \mathrm{~h}$, and the results of the paper show that the proposed method has relatively high accuracy. The paper analyzes the principle of EMUs brake control system, on the basis of which the key component is evaluated based on the Bayes theory under small sample, and then the system is evaluated based on the GO method. Finally, after estimating and calculating, we get parameter estimators of brake control system including reliability, MTBF and so on. The application of this method can achieve the purpose of estimating the reliability of the braking system to allow us to take measures to prevent accident occurring in advance, which has important research significance.

\section{CONFLICT OF INTEREST}

The authors confirm that this article content has no conflicts of interest.

\section{ACKNOWLEDGEMENTS}

The research work is supported by Natural Science Foundation of China under Grant no. 51175028.

\section{REFERENCES}

[1] D.X. Zhu and H.Z. Liu, "Reliability evaluation of high-speed train bearing with minimum sample", Journal of Central South University (Science and Technology), vol. 44, pp. 963-9, 2013.

[2] Y. Tian, Research on Extreme Small Sample Reliability Evaluation Method and its Application on Center Sill and Body Bolster of C70 Gondola Car, Beijing: Beijing Jiaotong University, 2008.

[3] J.L. Cao, Reliability Study of the Metro Traction Power Supply System Based on GO Methodology, Chengdu: Southwest Jiaotong University, 2012.

[4] M.N. Zhang, J.W. Yang and Q. Huang, "Reliability analysis of EMUs air source system based on GO method", Rolling Stock, vol. 52, pp. 31-4, 2014.

[5] Y.M. Li and W. Zhang, EMUs Brake Technology, Chengdu: Southwest Jiaotong University press, 2008.

[6] Z.H. Zhang, Reliability Theory and Engineering Application, Beijing: Science Press, 2012. 
[7] Z.M. Shen and X.R. Huang, GO method principle and application: A system reliability analysis method, Beijing: Tsinghua University press, 2004.

[8] J.Z. Dong, Study on urban rail vehicle brake system reliability modeling and simulation, Taiyuan: Taiyuan University of Science and Technology, 2012.

[9] M. Han, "The comprehensive estimation of failure rate under zero-failure data", Applied Mathematics A Journal of Chinese Universities, vol.17, no. 2, pp. 200-6, 2002.

[10] S.S. Mao and L.L. Wang, Reliability statistics, Shanghai: Higher Education Press, 1984.
[11] M. Han, "The synthesize hierarchical bayesian estimation of failure-rate of zero-failure data", Operations Research and Management Science, vol. 8, pp. 1-5, 1999.

[12] L.H. Xiong, Statistical analysis of reliability about zero-failure data, Changchun: Jilin University, 2004.

[13] Z.P. Shen, X.R. Huang and J Gao. "GO method application in reliability analysis of repairable system", Nulear Power Engineering, vol. 21, pp. 456-61, 2002.

Received: September 16, 2014

Revised: December 23, 2014

Accepted: December 31, 2014

(C) Chunqing et al.; Licensee Bentham Open.

This is an open access article licensed under the terms of the Creative Commons Attribution Non-Commercial License (http://creativecommons.org/licenses/by-nc/4.0/) which permits unrestricted, non-commercial use, distribution and reproduction in any medium, provided the work is properly cited. 